\title{
Designing Framework of Cloud Health Record (CHR) System for Patient Health Information Storage: A New Prophecy
}

Prakash Kuppuswamy ${ }^{1,}$, Shanmuga Sundaram ${ }^{2}$ and Rajan John ${ }^{3}$

${ }^{1}$ Department of CNET, College of Computer Science \& Information Systems, Jazan University, KSA

${ }^{2}$ Department of CS, College of Computer Science \& Information Systems, Jazan University, KSA

\begin{abstract}
Patient health record system offers significant health care benefits to improve the quality of health amenities, it has been reducing alarm rate of failures in medicinal treatment. Medical records contain large volumes of aggregated and longitudinal clinical data that could insist patient safety. In order to minimize the cost, replication of records and infrastructure, the emerging technologies like server virtualization and cloud computing is unavoidable at present medical environment. Cloud technology facilitates data storage, that keeps for all time availability of every individual patient information. Storing and maintaining the patient information is very essential for the future reference by any other hospitals and medical experts. Cloud computing makes convenient to the other stake holders of healthcare environment such as pharmacist, nurses, lab technicians, physician and other specialists. Existing patient's health records (PHR) maintained by manually and electronically (EHR) and stored in localized server controlled by hospital authority. In this article, we outlined new prophecy of Cloud based storing system called Cloud Health Record (CHR), we suggest that the new system will help to store huge volume of patient information and it will facilitate sharing the patient records on the cloud based system for getting further and better opinion from the physician, who lives anywhere.
\end{abstract}

\section{Introduction}

Patient health record is a paper repository of information that was reviewed or used for clinical, research, administrative and other government purposes. Paper based records accessed by single user only at a time. Records should be update every time by manual operation, resulting in delays for record completion that lasted anywhere from one to six months or more. Paper based records can be accessed and view by many people without any authorization, its triggered no safety and privacy [5]. Health information technology is evolving very fast and has penetrated in human lives that now it is absorbed in the system in a more promptly [4]. Many healthcare facilities from across the globe have implemented EHR systems to improve the information recording process but only a few have been successful [2,9]. Cloud environment provided centralized storage of data and connected remote server and it provides various data service accessed by remotely. Simply cloud computing is the acquisition to computing resources through the Internet [24-26].

Cloud computing is a kind of new environment which facilitates in sharing the resources and services over the internet rather than having these services and resources on local servers/ nodes or personal devices [1]. Cloud technology providing service as according to the usage of service, and it has raised more expectations. The significant benefits of cloud deployment reducing storage and maintenance of infrastructure cost [4]. Cloud offers great opportunity for medical related application to connect their services not only promptly but also in new formats with the flexibilities such data storing, editing, modification, exchange, access from any places. Many hospitals are planning to build and their own data centers to storing the patient information for sharing the information to other hospitals or future references.

The new concept of cloud and patient health record has generated a new model called Patient cloud records. The usages of cloud computing may vary with the medical application such as nature, services and information needs but most common usages of cloud computing with in medical application can be development of patient records and information storing on the cloud. Cloud-based environment system can offer provides several advantages such as scalability, availability and mobility $[4,7-10]$, and there are some barriers that must also be managed $[4,15,16]$. Establishing Cloud-based EHR management system, the main advantage is the ability to share patient records with other clinical centers, and the integration of all the EHRs of a group of clinical centers in order to help medical staff perform their jobs $[4,17-$ 19]. The reason for offering cloud computing technology in Patient health records, it provides basic infrastructure to hosting remotely the large volume of patient information and it can be accessed by anyone directly over the internet. The main objective of Health Records to support the maintenance of integrated, efficient and quality health $[23,24]$. To establish efficient cloud structure, we need following three services:

1. Software as a service (SaaS) required hardware, networking software and operating system are provided by the service.

2. Platform as a service (PaaS) need Operating system, networking software and hardware is provided by the service provider, while the client has to manage its required software and applications.

3. Infrastructure as a service (IaaS) need only the hardware and network are provided by the service. The users have to manage for their operating system, software and applications.

"Corresponding Author: Dr. Prakash Kuppuswamy, Department of CNET, College of Computer Science \& Information Systems, Jazan University, KSA E-mail: prakashcnet@gmail.com

Citation: Kuppuswamy P, Sundaram S, John R (2020) Designing Framework of Cloud Health Record (CHR) System for Patient Health Information Storage: A New Prophecy. Int J Comput Softw Eng 5: 153. doi: https://doi.org/10.15344/2456$4451 / 2020 / 153$

Copyright: (c) 2020 Kuppuswamy et al. This is an open-access article distributed under the terms of the Creative Commons Attribution License, which permits unrestricted use, distribution, and reproduction in any medium, provided the original author and source are credited. 
Citation: Kuppuswamy P, Sundaram S, John R (2020) Designing Framework of Cloud Health Record (CHR) System for Patient Health Information Storage: A New Prophecy. Int J Comput Softw Eng 5: 153. doi: https://doi.org/10.15344/2456-4451/2020/153

Page 2 of 5

Cloud technology enabling on-demand network access to shared pool of computing resources and accessible by anytime and anywhere. Therefore, the organization of National Institute of Standards and Technology (NIST) defines standard essential characteristics of cloud computing. This cloud computing model is composed of three service model, four deployment models and following six essential characteristics.

1. On-demand self-service: In this set up the resources are provided to the user on demand. The user has option to manage these computing resources.

2. Resource pooling: In this setup, the resources made available from a shared pool of data.

3. Broad network access: In this set up services are available over internet and/or over a private network.

4. Rapid elasticity: In this set up extent of services can be scaled as per the requirement in what is called as horizontal or vertical scaling technique.

5. Measured service: This setup is something like metered services. The user pays only for the extent he uses the services.

6. Multi Tenacity: It is new characteristics, refers the policy-driven enforcement, segmentation, isolation, governance, service levels. User's might utilize a public cloud provider's service offerings [28].

\section{Literature Review}

Repu Daman, Manish Tripathi, Saroj Mishra (2016) discussed about using of mobile computing and cloud technology in healthcare domain. Maintaining the secrecy of patients' records and information is responsible of hospital management and that record helps the quality of treatment. The application of cloud computing reduces cost and all time availability of patient's record. In addition, authors elaborated about the advantage of cloud data access that can be available anywhere in the geographical region. They suggest hospitals and other healthcare providers need to adapt quickly to the cloud technologies and collaborate more efficient manner to improve the quality of service in health industry [4].

Elise Russo, Dean F. Sittig, Daniel R. Murphy, Hardeep Singh (2016) Electronic health record (EHR) data storage consist huge volume of data. Keeping patient information or data safety successes by few software model. In this research article author analyzed with a model of missed/delayed follow-up of test results to discuss realworld challenges in using EHR data for patient safety research. The output of the research brought three types of challenges that pose as barriers to advance patient safety improvement. Author identifies problems of existing health record maintenance. Health records and their abundant data for patient safety improvement research, many current data access and security policies and procedures must be rewritten and standardized across health care organizations [6].

Ebru Celikel Cankaya, Than Kywe, (2015) In this article author prosed comprehensive electronic health record system based on AES encryption standard. Proposed database model using as a software engineering approach and database development model and combine the two to present a guideline to design and develop other similar projects. Data based model design with ER model and using $3 \mathrm{NF}$ normalized form.

Int J Comput Softw Eng

ISSN: 2456-4451
This proposed model can be extending design to provide an interactive tool that will help user get involved actively in the design process [7].

Pradeep Deshmukh (2017) Discussed about significance of health care system used by its stake holder of healthcare user such as nurses, specialists and patient. Cloud provide low cost and flexible to save patient health records with data privacy. Author proposed frame work of saving the health records and provides the accessibility to the physicians, patients and other healthcare users by providing accessible key. It provides the security to the health records with data encryption and stored data. Proposed model integrate rural and urban health care centers and hence more appropriate for health care services. The experimental result shows that it has a capability of scaling more number of records with effective safety and security [8].

Jaillah Mae Gesulgaa, Almarie Berjameb, Kristelle Sheen Moquialac (2017) Moreover, this article reviews and identifies barriers of EHR system. Authors proposed structured model of health record system. It's identifies problems of Health record system depends on user's lack of education and training, and concerns arising from data security were the predominant barriers recognized from the analyzed papers. In addition to that, author directed to examine the barriers in detail and recognize [9].

Everleen Wanyonyi, Anthony Rodrigues, Silvance Abeka, Solomon Ogara (2017) This study focused on the EHR security and threats. Author took sample questionnaire designed for qualitative data collection. This research article identified controlling and securing patient health record not possible by single control system. The system required physical security and software security and technical and administrative controls. The study established the effect of availability of resources and organizational culture on EHR information security, though most of the characteristics or factors of the two moderating variables were already covered in the predictor variables and therefore were not analyzed separately [10].

\section{Significance of Research}

Adoption of advance technology such as Cloud computing, Mobile computing and Big data analytics is far behind in Medical and applied medical sector. Primary work of healthcare institutions and hospitals depends on patient health records such as medical prescription and reports in a form of film-based radiological \& pathological images, handwritten patient diagnosis and patient history etc., Collecting and keeping the above information and records are very tedious work for the Medical Record Department. The collaboration with the other hospitals and intra-communication between different departments, wards and Outpatient clinics are really challenging task. Customary Medical Record system requires entire infrastructure to storing and arranging data and it requires manpower to collecting information. Unified computing model is a vendor-designed system, more cost and centrally managed by the data centre and it is suitable for only big size hospital.

Medical practitioner is looking an integrated health records of patient that will provide a global and complete opinion of a patient health history that can lead to a better decision support by physicians during medical emergency or general consultation. Existing Grid computing based virtual environment also supports to store the large volume of data. But, it causes great pressure on the Electronic Health record server; also it leads transmission delays due to the network 
Citation: Kuppuswamy P, Sundaram S, John R (2020) Designing Framework of Cloud Health Record (CHR) System for Patient Health Information Storage: A New Prophecy. Int J Comput Softw Eng 5: 153. doi: https://doi.org/10.15344/2456-4451/2020/153

Page 3 of 5

traffic. There may be a possibility of data delays or data loss due to the physical network accessories can have serious consequences that cannot be measured. Cloud based health record system has a significant change in providing healthcare information, reducing errors and increasing impact of health care. Accessing of patient history may provide better treatment and it improves more patient safety.

\section{Proposed Structure and Implementation Plan}

Keeping large volume of electronic information such as documents, images, emails, videos in organizations produce is staggering. Moreover, storing all your digital data in your data center can be expensive. Cloud storage supports to solve the above said problems with nominal maintenance cost. Internet providing facility of connecting cloud user can access and update data from anywhere and anyplace. It supports easy handling and storing patient information on remote networks.

This study aimed to enhance the facility of better administration and access of patient health information through the cloud storage. Existing storage system is not fulfilling the objective and effectiveness of patient health information on cloud storage. In general, the health care services are divided as Urban Health center, Rural Health Centers and Primary Health centers. Comparing to City and Rural, primary health services will be very basic and less advanced. The treatment with specialized health services in physician and equipment point of view will not be available in most parts of the rural and primary area. The significant advantage of implementing Cloud Heal Records (CHR) are connecting Primary Health Center, Rural Health center and Urban Health center directly through cloud medical server and it will be providing better service to patient. Proposed system architecture showed in figure 1, we are trying to provide complete and better utilization of cloud model patient health record system and privacy. The proposed method authorization given to many clinical persons to enter and update patient information. Cloud Health Records (CHR) segregated into patient details consist basic information of individual person. and next patient history consist of previous health issue details of patient and third one consist of sensitive information such as cancer, HIV and similar disease history.

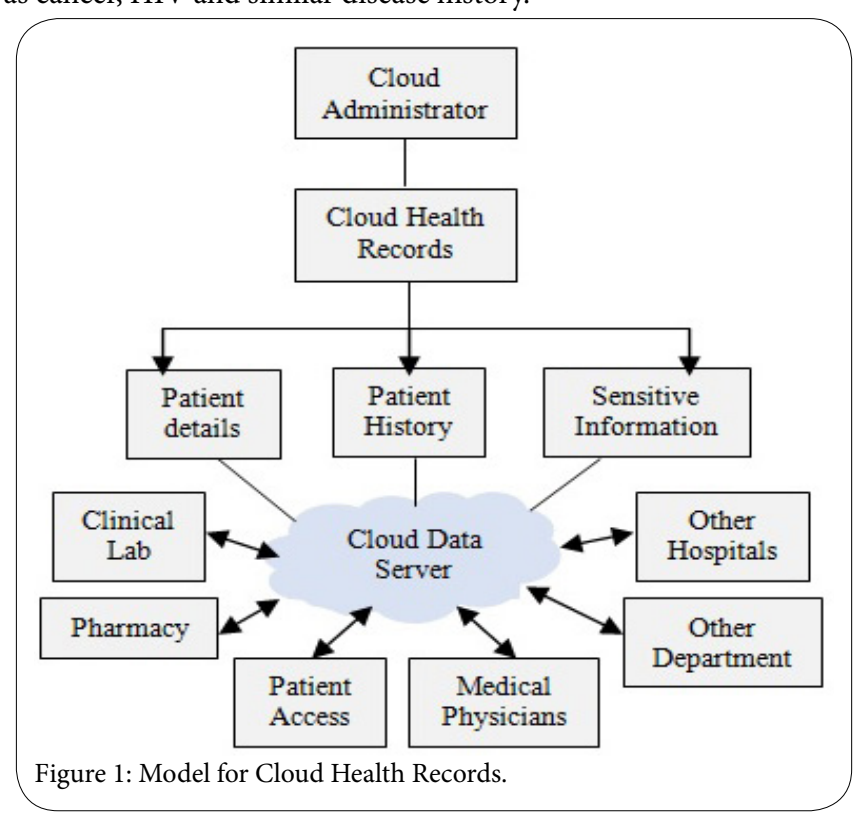

Int J Comput Softw Eng

ISSN: 2456-4451
CHR transactions are conducting through central hub is called Cloud Data server and its controlled by Cloud administrator. The Centre hub of Cloud Data storage access granting by the cloud administrator to Physician, Clinical Lab, Pharmacy, other department, other hospital, patient and government for upload records and updating information. All above users/patients can enter their own personal health record data in this database. The CHR database consists of a vast number of patient health care records containing sensitive information. The technical part of cloud administration controls uses software and data to monitor and control access to information and computing systems. Responsibility of cloud administrator controls include passwords, firewalls, network intrusion detection systems, access control lists and data privacy.

\section{Responsibility of Cloud Administrator}

Cloud administrator works defined as management controls of written policies, procedures, standards and guidelines etc., Cloud Administrator controls inform users on how the data is to be access, save and protect the sensitive data. Cloud admin has to follow government laws and regulation, hospital policy, hiring policy, password policy and disciplinary policy etc., The significant work of Cloud Health Records mentioned in the above figure to controls patient basic information, patient history and sensitive information maintenance. The summarized points mentioned below:

\section{Identify and track the cloud server patient's data}

2. Create accessible policy to the Government, Physician, Clinical person, patient and other hospital.

3. Concede and confirm the patient details uploaded by the physician for resource sharing

4. To improve technological skills

5. To provide technical and software support to the user

6. To conduct training and awareness programs for users

7. To provide inter-department data sharing facility

8. To create strategic planning and decision making ability to provide accessibility

9. To create forum for open discussion with patient and medical experts

10. Identify usage record of cloud health records

\section{Benefits of the Proposed Model}

Patient information sharing Service: Cloud platform is now becoming popular to offer the patient information access to their collection on one platform.

Health record Download Service: One can download records/digital images/documents easily if permit access in the cloud network.

Digital conservancy: Digitization task took centralized hub and it can avoid time consuming to duplicate the data. Cloud data storage can preserve the collection is digital form in the form of archives.

Medical Report Delivery Service: Cloud computing can be used for medical report delivery service to the patients at their home through mobile service. 
Citation: Kuppuswamy P, Sundaram S, John R (2020) Designing Framework of Cloud Health Record (CHR) System for Patient Health Information Storage: A New Prophecy. Int J Comput Softw Eng 5: 153. doi: https://doi.org/10.15344/2456-4451/2020/153

Page 4 of 5

Awareness Service: To provide current awareness service to all users has become easy with cloud computing.

Cost: Cloud technology providing centralized environment and it reduce cost of buying hardware and software and setting up.

Announcement service: We can provide new services of announcement or guidelines on bulletin board with this Cloud technology.

Collection Development: Cloud computing is used for collection development that it can avoid duplicate resources to physician and other users.

Information Discovery: Cloud provides a platform to store all information/records/images that one can access anytime from anywhere; so information discovery and searching become easy and it is very useful for authorized user.

Health Diagnostic Learning: Diagnostic symptoms and relevant material can be kept on the cloud for reference purpose and online examinations also can be conducted. Further, Consultations, alterations can be done at a time from various locations.

Health records case study: Cloud Health Record system (CHR) provides the facility of keeping reports, medical images and videos, presentations and medical data on the cloud for user's orientation.

Global Scale: CHR services include the ability to scale elastically. It can provide the facility to connect global specialist to sharing their experience and knowledge.

Performance: CHR services run on a worldwide network of secure datacenters, which are regularly upgraded to the latest generation of fast and efficient computing hardware. This offers several benefits over a sharing medical resources effectively.

Reliability: CHR system provide the facility of data backup, disaster recovery services at multiple redundant sites on the cloud network.

\section{Comparison Analysis}

Different scenarios involve medical records, but with one differenceone type is considered paper medical records, Electronic Medical Records, Electronic Health Records. Paper records require additional personnel to handle and support paper files and to organize countless documents. Other modern health record comparison mentioned in the below table 1 .

Digitalization of records can save emergency care providers time during a patient's visit. One of the most obvious benefits of going digital is the reduction of adverse environmental impacts, Cloud based health record system provides numerous cybersecurity and data breaches. It's trying to reduce human administrative costs and storage costs. Sharing patient's data is much easier; patients and medical personnel can access information via an app or by sending a photo via a secured network. Cloud based record management costs by reducing on premise hardware and software management, simplifying monitoring, and reducing the need for extensive capacity planning. Many cloud storage service provided the facility of keeping multiple copies of patient data, and offer great object durability to avoid data loss.

\section{Conclusion}

Technology is advancing at a phenomenal rate, but it needs to be harnessed in the most efficient way. Cloud technology, resulting in cloud health records will certainly provide a great leap forward in providing access to large quantities of information to users. Cloud providing huge volume of data storage service to keep various data scheme such as document, image, gif and videos. Currently, standard and specific cloud application available in the market, it is provided by many cloud service companies. Some of the well-known cloud services are drop box, Sugar Sync, Apple iCloud, Google Drive, Microsoft OneDrive etc., Standard cloud service offers real-time document co-editing and these products even double as collaboration software. Proposed Cloud Health Record facilitates various services such as backup of health details and keeping availability of records in-order to any disk failures. Backup services always safer and it is

\begin{tabular}{|c|c|c|c|}
\hline & $\begin{array}{l}\text { EMR } \\
\text { Electronic Medical Records }\end{array}$ & $\begin{array}{l}\text { EHR } \\
\text { Electronic Health Records }\end{array}$ & $\begin{array}{l}\text { CHR } \\
\text { Cloud Health Records }\end{array}$ \\
\hline Cost & Less-suitable for small scale sector & High and suitable for multi-specialty Hospital & $\begin{array}{l}\text { High, suitable for super specialty } \\
\text { hospital }\end{array}$ \\
\hline Data process & Average-upgradation required & $\begin{array}{l}\text { Good-upgrade support provide by server } \\
\text { admin }\end{array}$ & $\begin{array}{l}\text { Excellent-upgradation provide by } \\
\text { cloud vendor }\end{array}$ \\
\hline Medical data & Text based result storage & Text and image based storage & All formats can store and transfer \\
\hline Environment & Access within clinic & Complete data, partially Accessible by others & Access anywhere, any time \\
\hline Quality of service & Low & Medium & High \\
\hline Data entry & Slow & Fast & Fast \\
\hline Remote monitoring & Not Available & Available & Available \\
\hline Transparency & No & Yes & Yes \\
\hline Patient Access & No & Yes & Yes \\
\hline Server & Local server & Local/Cloud vendor & Cloud based server \\
\hline Data Backup & Manual backup by admin & Data available in local and cloud vendor & Data available in multiple place \\
\hline Security & $\begin{array}{l}\text { Not secured, possibility of malware } \\
\text { attack }\end{array}$ & $\begin{array}{l}\text { Secured, Malware protection support by } \\
\text { vendor }\end{array}$ & $\begin{array}{l}\text { More secured, Multiple protection } \\
\text { support by Cloud admin }\end{array}$ \\
\hline
\end{tabular}

Table 1: Health record service comparison. 
Citation: Kuppuswamy P, Sundaram S, John R (2020) Designing Framework of Cloud Health Record (CHR) System for Patient Health Information Storage: A New Prophecy. Int J Comput Softw Eng 5: 153. doi: https://doi.org/10.15344/2456-4451/2020/153

Page 5 of 5

accessible by only cloud administrator. Cloud health record service can extend and access from all geographical location. The proposed structure model is giving suggestion to improve the patient health information to store and accessibility through Cloud environment. This work is an initiation of organizing patient information on Cloud based storage in such a manner and it can be extending in future work with security aspects of the records.

\section{Competing Interests}

The authors declare that they have no competing interests.

\section{References}

1. Kaushik A, Kumar A (2013) Application of cloud computing in libraries International Journal of Information Dissemination and Technology 3: 270273.

2. Hasanain RA, Cooper H (2014) Solutions to overcome technical and social barriers to electronic health records implementation in Saudi public and private hospitals. J Health Inform Dev Ctries 8: 46-63.

3. Rodrigues JJ, de la Torre I, Fernández G, López-Coronado M (2013) Analysis of the Security and Privacy Requirements of Cloud-Based Electronic Health Records Systems. J Med Internet Res 15: 186

4. Daman R, Tripathi MM, Mishra SK (2016) Cloud Computing for Medical Applications \& Healthcare Delivery: Technology, Application, Security and Swot Analysis. ACEIT Conference Proceeding.

5. Harman LB, Flite CA, Bond K (2012) Electronic Health Records: Privacy Confidentiality, and Security. Journal of Ethics. Illuminating the Art of Medicine.

6. Russo E, Sittig DF, Murphy DR, Singh H (2016) Challenges inpatient safety improvement research in the era of electronic health records. Healthc 4 : 285-290

7. Cankaya EC, Kywe T (2015) A Secure Healthcare System: From Design to Implementation. International Conference on Soft Computing and Software Engineering SCSE, Procedia Computer Science 62: 203-212.

8. Deshmukh P (2017) Design of cloud security in the EHR for Indian healthcare services. Journal of King Saud University Computer and Information Sciences 29: 281-287.

9. Gesulgaa JM, Berjameb A, Moquialac KS (2017) Barriers to Electronic Health Record System Implementation and Information Systems Resources: A Structured Review, Adrian Galidod, 4th Information Systems International Conference, ISICO.

10. Wanyonyi E, Rodrigues A, Abeka S, Ogara S (2017) Effectiveness of Security Controls on Electronic Health Records. International Journal of Scientific \& Technology Research.

11. Furth B, Escalante A (2010) Handbook of Cloud Computing. 1st Edition. London: Springer.

12. Chen YY, Lu JC, Jan JK (2012) A secure EHR system based on hybrid clouds. J Med Syst 36: 3375-3384.

13. Low C, Hsueh Chen Y (2012) Criteria for the evaluation of a cloud-based hospital information system outsourcing provider. J Med Syst 36: 35433553.

14. Poulymenopoulou M, Malamateniou F, Vassilacopoulos G (2012) Emergency healthcare process automation using mobile computing and cloud services. J Med Syst 36: 3233-324.

15. Buyya R, Ranjan R (2010) Special section: Federated resource management in grid and cloud computing systems. Future Generation Comput Syst 26: 1189-1191.

16. Bateman A, Wood M (2009) Cloud computing. Bioinformatics 25: 1475

17. Armbrust M, Fox A, Griffith R, Joseph AD, Katz R, et al. (2010) A view of cloud computing. Commun ACM 53: 50-58.

18. Kuo AM (2011) Opportunities and challenges of cloud computing to improve health care services. J Med Internet Res 13: 67.

19. Anderson NR, Lee ES, Brockenbrough JS, Minie ME, Fuller S, et al (2007) Issues in biomedical research data management and analysis: needs and barriers. J Am Med Inform Assoc14: 478-488.
20. Svantesson D, Clarke R (2010) Privacy and consumer risks in cloud computing. Comput Law Secur Rev 26: 391-397.

21. Fernández-Cardeñosa $G$, de la Torre-Díez I, López-Coronado $M$, Rodrigues $J$ (2012) Analysis of cloud-based solutions on EHRs systems in different scenarios. J Med Syst 36: 3777-3782.

22. Fernández-Cardeñosa G, De la Torre-Díez I, Rodrigues JJ (2012) Analysis of the Cloud Computing Paradigm on Mobile Health Records Systems. In: Proceedings of the Sixth International Conference on Innovative Mobile and Internet Services in Ubiquitous Computing, Italy.

23. Schloeffel P (2002) Electronic Health Record Definition. Scope and Context. ISO. TC.

24. Ahmadi M, Aslani N (2018) Capabilities and Advantages of Cloud Computing in the Implementation of Electronic Health Record. Acta Inform Med 26: 24-28.

25. Buyya R, Yeo CS, Venugopal S, Broberg J, Brandic I, et al. (2009) Cloud, computing and emerging IT platforms: Vision, hype, and reality for delivering computing as the 5 th utility. Future Generation computer systems 25: 599-616.

26. Kanagaraj G, Sumathi AC (2011) Proposal of an open-source Cloud computing system for exchanging medical images of a Hospital Information System. In Trends in Information Sciences and Computing (TISC), IEEE 3rd International Conference.

27. Armbrust M, Fox A, Griffith R, Joseph AD, Katz R, et al. (2010) A view of cloud computing. Communications of the ACM 53: 50-58.

28. https://www.isaca.org/Groups/Professional-English/cloud-computing/ GroupDocuments/Essential characteristics of Cloud Computing.pdf 